\title{
Investigating the relationship of DNA methylation with mutation rate and allele frequency in the human genome
}

\author{
Junfeng $\mathrm{Xia}^{1 \dagger}$, Leng $\mathrm{Han}^{1 \dagger}$, Zhongming Zhao $1,2,3,4^{*}$
}

From The International Conference on Intelligent Biology and Medicine (ICIBM)

Nashville, TN, USA. 22-24 April 2012

\begin{abstract}
Background: DNA methylation, which mainly occurs at $\mathrm{CpG}$ dinucleotides, is a dynamic epigenetic regulation mechanism in most eukaryotic genomes. It is already known that methylated CpG dinucleotides can lead to a high rate of $C$ to $T$ mutation at these sites. However, less is known about whether and how the methylation level causes a different mutation rate, especially at the single-base resolution.

Results: In this study, we used genome-wide single-base resolution methylation data to perform a comprehensive analysis of the mutation rate of methylated cytosines from human embryonic stem cell. Through the analysis of the density of single nucleotide polymorphisms, we first confirmed that the mutation rate in methylated CpG sites is greater than that in unmethylated $\mathrm{CpG}$ sites. Then, we showed that among methylated $\mathrm{CpG}$ sites, the mutation rate is markedly increased in low-intermediately (20-40\% methylation level) to intermediately methylated CpG sites (40-60\% methylation level) of the human genome. This mutation pattern was observed regardless of DNA strand direction and the sequence coverage over the site on which the methylation level was calculated. Moreover, this highly non-random mutation pattern was found more apparent in intergenic and intronic regions than in promoter regions and $\mathrm{CpG}$ islands. Our investigation suggested this pattern appears primarily in autosomes rather than sex chromosomes. Further analysis based on human-chimpanzee divergence confirmed these observations. Finally, we observed a significant correlation between the methylation level and cytosine allele frequency.
\end{abstract}

Conclusions: Our results showed a high mutation rate in low-intermediately to intermediately methylated CpG sites at different scales, from the categorized genomic region, whole chromosome, to the whole genome level, thereby providing the first supporting evidence of mutation rate variation at human methylated CpG sites using the genome-wide sing-base resolution methylation data.

\section{Background}

Germline mutation can profoundly affect genetic variation within populations and divergence between species. The identification of factors that modulate mutation rates in germ cells, particularly in humans, has been a long-standing scientific pursuit [1]. Several factors are thought to be related to mutation rate, including DNA

\footnotetext{
* Correspondence: zhongming.zhao@vanderbilt.edu

+ Contributed equally

'Department of Biomedical Informatics, Vanderbilt University School of Medicine, Nashville, TN 37232, USA

Full list of author information is available at the end of the article
}

replication timing [2], genetic recombination [3], and categorized genomic regions [4]. The mutation rate is also known to vary throughout the genome in a contextual fashion [1], the most well-studied example being the influence of $\mathrm{CpG}$ dinucleotides in mammalian genomes. CpGs are generally methylated in mammalian genomes, and since the deamination rates of methylated cytosine is 2.0 to 3.2 fold more than unmethylated cytosine [5], it leads to a 10 - to 50 -fold higher transition rate of methylated CpG to TpG than other transitional changes [6,7]. This rate difference in turn causes the suppression of CpG sites in the mammalian genomes [8,9]. However,
C Biomed Central

() 2012 Xia et al.; licensee BioMed Central Ltd. This is an open access article distributed under the terms of the Creative Commons Attribution License (http://creativecommons.org/licenses/by/2.0), which permits unrestricted use, distribution, and reproduction in any medium, provided the original work is properly cited. 
whether and how a variation in methylation level causes the variation in the high mutation rate is still unknown.

Based on the analysis of single nucleotide polymorphisms (SNPs), Fryxell and Moon [10] have concluded that methylation-dependent transition rates are exponentially dependent on local GC content. Our re-examined studies further indicated that these methylation-dependent transition rates in the human genome were dependent on both the local sequence length and the genomic region [7]. In a recent study of CPG mutability in intronic regions of the human genome [11], CpG substitution rates were observed to be significantly correlated with the extent of methylation level. In the same study, they also found that the CpG substitute rate is positively correlated with non- $\mathrm{CpG}$ divergence and negatively correlated with GC content [11]. However, their conclusion is based on MeDIP methylation data, which is calculated in a low resolution for a 100-bp (base pair) window size [12]. Moreover, although their results showed that the methylation level affected the mutation rate, their investigation limited in the intronic regions only, and the mutation pattern in other genomic regions remained unclear.

More recently, next-generation sequencing has provided single-base resolution DNA methylome in the human genome [13], thus making more comprehensive analysis possible [14-17]. The availability of whole-genome methylation maps with single-nucleotide resolution provides an opportunity to refine studies pertaining to CpG mutation rate variation. Compared with array data, the advantage of sequencing data can not only be used to estimate methylation broadness but also be used to analyze methylation deepness for each single base [17].

Here, we used genome-wide, single-base-resolution maps of methylation data [13] in human embryonic stem cell line $(\mathrm{H} 1)$ to perform an extensive investigation of the correlation between the level of cytosine methylation and CpG mutability of the human genome. In addition to the methylation on CpG sites, we also examined the effect of methylation level on non-CpG sites (e.g., $\mathrm{CHG} / \mathrm{CHH}$, where $\mathrm{H}=\mathrm{A}, \mathrm{C}$, or $\mathrm{T}$ ) sites. We observed that among methylated CpG sites, the mutation rate, as reflected by the human SNP density, is markedly increased in lowintermediately to intermediately methylated $\mathrm{CpG}$ sites at different scales, from the categorized genomic region, whole chromosome, to the whole genome level. This observation was also confirmed by human-chimpanzee divergence data. Finally, we found a significant correlation between methylation level and cytosine allele frequency in the human genome.

\section{Materials and methods}

\section{DNA methylation profile in the human genome}

The single-base resolution DNA methylation data were derived from Lister et al. [13], including whole genome bisulfite sequencing data for two human cell lines: $\mathrm{H} 1$ human embryonic stem cell and IMR90 fetal lung fibroblast. We removed all methylcytosines that could not be mapped to reference CpG sites (NCBI Build 36) in our analysis. For the remaining mapped methylcytosines, we used methylation deepness [17] to evaluate the methylation level in the CpG site, which represents the extent of the methylation level of the cytosines based on the ratio of the number of reads having methylated cytosine over the total number of reads mapped to each reference $\mathrm{CpG}$ site. CpG sites can be classified as methylated CpG sites if there is at least one read covering methylated cytosine (methylation level $>0 \%$ ). Otherwise, they are classified as the unmethylated $\mathrm{CpG}$ sites, i.e. methylation level $=0 \%$. Methylated CpG sites are further categorized into five groups of increasing methylation levels: lowly methylated (less than or equal to $20 \%$ ), low-intermediately methylated (20-40\%), intermediately methylated (40-60\%), high-intermediately methylated (60-80\%), and highly methylated (greater than 80\%).

\section{Genome sequence and annotation}

The human reference genome sequence (Build 36) was downloaded from NCBI website [18]. Genomic annotations, including the start and end positions of genes and exons, were defined based on the ENSEMBL database [19]. Promoter was defined as an interval of -1500 to +500 bp around the transcriptional start site (TSS) as previously described[20]. A genic region was defined as the transcribed region from the start to the end of transcription sites. An intergenic region was defined as a region in which no known gene was annotated. Similarly, an intronic region was defined as the region in which no known exon was annotated [20]. According to our previous comparisons [21,22], we used Takai and Jones' algorithm [23] to identify CpG islands (hereafter, we abbreviated as CGIs): length $\geq 500 \mathrm{bp}, \mathrm{GC}$ content $\geq 55 \%$, and $\mathrm{CpG}$ observed/expected $(\mathrm{O} / \mathrm{E})$ ratio $\geq 0.65$.

\section{Estimation of mutation rate}

To estimate the mutation rates, we followed the approach in previous studies $[2,24]$ to use the data from both human SNP density and human-chimpanzee divergence. We downloaded the human SNPs data from the NCBI dbSNP database (build 130) website [18] and only considered the validated reference SNPs [4]. A total of $16,969,034$ validated reference SNPs were available (with reference SNP ID and reference annotation). Among them, 12,236,967 were bi-allelic and uniquely mapped in the genome, which were used for our further analysis. The SNP density is estimated by computing the total number of $\mathrm{C} / \mathrm{A}, \mathrm{C} / \mathrm{G}$, and $\mathrm{C} / \mathrm{T}$ SNPs over the total number of cytosine bases in the human genome. 
We estimated the divergence of CpGs between the human and chimpanzee genomes based on the human and chimpanzee alignment. We downloaded alignments of the hg18 version of the human genome and PanTro2 version of the chimpanzee genome from UCSC Genome Browser [25]. The divergence is calculated as the proportion of different sites between human and chimpanzee genomes among the total sites that could be aligned between human and chimpanzee genomes.

\section{Human allele data}

For human allele frequency data, we used CEU (Utah residents with Northern and Western European ancestry from the CEPH collection) population data that we downloaded from UCSC Genome Browser [25]. This data set also includes chimpanzee nucleotide information for each allele set when available; thus, we could determine which allele was the ancestral one based on our previous study[4].

\section{Results and discussion}

\section{Mutation rate in CpG sites with different methylation} level

To investigate the mutation rates of $\mathrm{CpG}$ sites with different methylation levels, we analyzed the SNP density in the human genome, which represented recent mutations in the human population [2]. We mapped C/A, C/G, and C/
T SNPs to CpG sites regardless of their methylation levels. For CpG sites within the same methylation level group, we estimated the mutation rate by computing the number of these SNPs $(\mathrm{C} / \mathrm{A}, \mathrm{C} / \mathrm{G}$, and $\mathrm{C} / \mathrm{T})$ over the total number of cytosine bases in the human genome. Since methylated CpGs are highly mutable, one would expect to observe that CpGs with high levels of methylation are more likely to mutate. Indeed, we found that the unmethylated CpGs had a lower mutation rate $(1.08 \%)$ compared with methylated CpGs (3.55\%) (Figure 1). Here, mutation rate was measured by the SNP density [2]. The mutation rate in methylated CpGs was also slightly higher than the total CpGs (3.16\%) in the human genome, since more than $80 \%$ of CpGs are methylated in the human genome [13]. Among the five $\mathrm{CpG}$ methylation level groups, we observed a trend that when $\mathrm{CpG}$ methylation level increased, the proportion of $\mathrm{CpG}$ sites in that group increased as well (the classification of methylation level was described in the Materials and Methods section). Specifically, the number of $\mathrm{CpG}$ sites with lowly, low-intermediately, intermediately, high-intermediately and highly methylated levels was 168,353 (0.7\%), 511,909 (2.2\%), $1,384,329$ (5.9\%), 4,557,575 (19.5\%), and 16,788,938 (71.7\%), respectively. Interestingly, when we compared mutation rate in different groups of methylated CpGs, we found that CpG sites with low-intermediate (20-40\%) and intermediate (40-60\%) levels of methylation actually had a

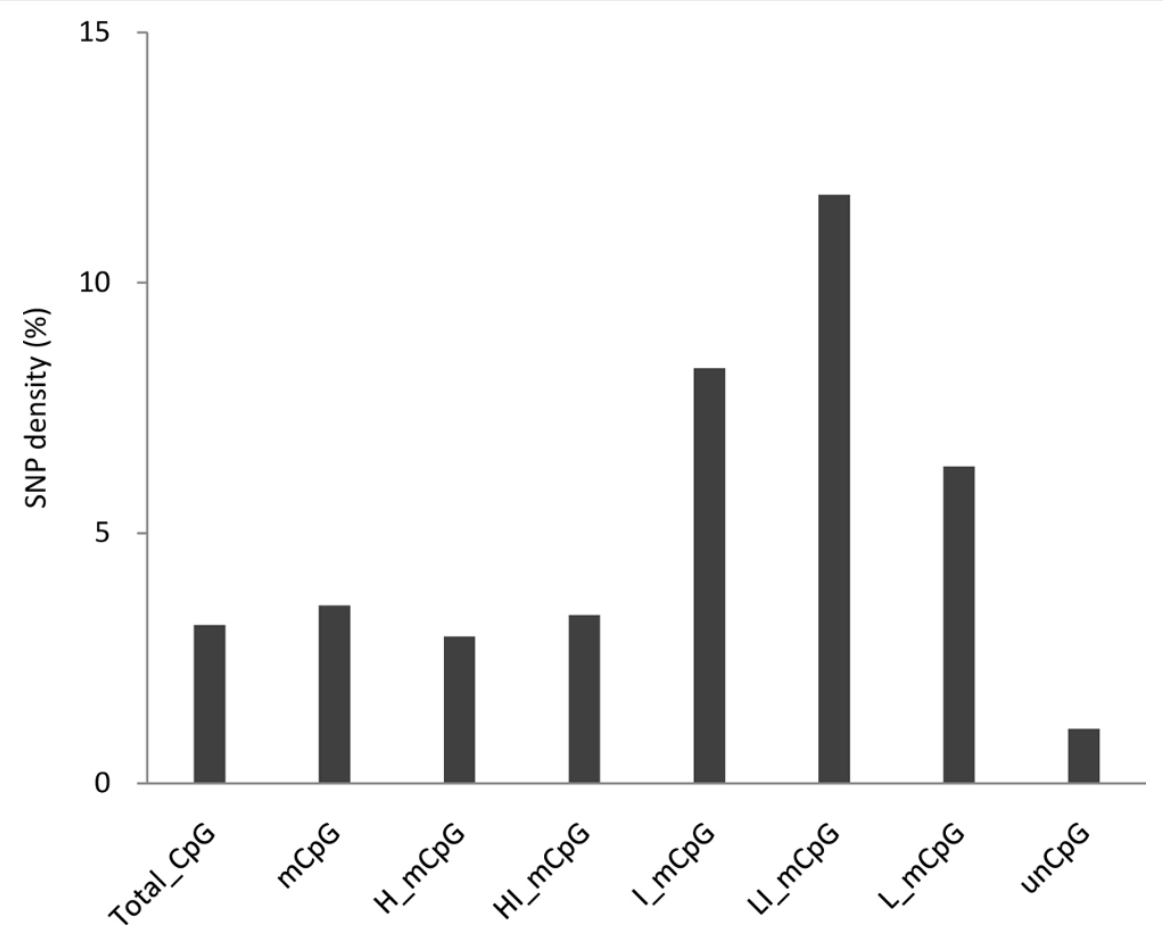

Figure 1 The mutation rates for all CpG sites, methylated CpG sites and unmethylated CpG sites. The methylated CpG sites are further categorized into five groups according to their methylation levels. The X-axis includes CpG sites with different methylation levels (Total_CpG: total CpG; mCpG: methylated CpG; H_mCpG: highly methylated CpG (> 80\%); HI_mCpG: high-intermediately methylated CpG (60-80\%); I_mCpG: intermediately methylated CpG (40-60\%); LI_mCpG: low-intermediately methylated CpG (20-40\%); L_mCpG: lowly methylated CpG ( $\leq 20 \%)$; unCpG: unmethlated $(p G)$. The Y-axis is the mutation rate that was calculated as the SNP density at CpG sites. 
much higher mutation rate compared with lowly $(\leq 20 \%)$, high-intermediate (60-80\%) and highly methylated CpGs (> 80\% methylation). For example, low-intermediately methylated CpG sites had a high mutation rate that could reach up to $11.75 \%$, whereas highly methylated and lowly methylated CpGs only had a mutation rate of $2.93 \%$ and $6.32 \%$, respectively.

We compared the mutation rate of methylated CpG sites in $\mathrm{H} 1$ and IMR90 cell lines (see additional file 1). We found that the mutation pattern in IMR90 cell line is overall similar to that in $\mathrm{H} 1$ cell line, though it is not as evident as in $\mathrm{H} 1$ cell line. One possible reason is that the $\mathrm{H} 1$ cell line is derived from human embryonic stem cells while the IMR90 cell line is originated from human fetal lung fibroblasts. It has been suggested that stem cells have more opportunity to accumulate mutations compared with mature cells [26]. Therefore, the mutation pattern shows stronger difference in CpG sites with 20-60\% methylation level in $\mathrm{H} 1$ cell line than IMR90 cell line.

To examine whether the strand bias affected the mutation pattern, we further analyzed the data in the minus strand to compensate for the plus strand pattern observed above. We observed the same pattern when the methylation data of the minus strand was analyzed (see additional file 2). This observation is consistent with the previous report that the DNA methylation levels of two strands in any given genomic region are highly symmetric [13].

The methylation profile we used in this study was generated by single-base resolution bisulfite sequencing technology. According to this technology, the more reads that cover the CpG site, the higher confidence we can obtain regarding the base it detects. Therefore, we checked whether our observation was affected by the read coverage. We found an overall similar pattern regardless of the cut-off values of the coverage we used (see additional file 3). Particularly for CpG sites with low-intermediate and intermediate levels of methylation, we found an even higher mutation rate with higher coverage. For example, we observed that the mutation rate increased to $15.26 \%$ when intermediately methylated $\mathrm{CpG}$ sites were covered by at least 10 sequencing reads compared with $11.00 \%$ when they were covered by at least 5 reads. This finding suggests that our observation that $\mathrm{CpG}$ sites with lowintermediate and intermediate levels of methylation actually had a much higher mutation rate is not affected by the sequence coverage of methylated sites. With the increased coverage, our observations could be even more apparent.

\section{Mutation rate of methylated CpG site in the categorized genomic regions}

We previously observed the correlation of the rate of germ line mutation with the categorized genomic regions [4]. Here, we further investigated the mutation rates of methylated CpG sites in categorized genomic regions. The results (see Figure 2) are summarized in the following four points. First, we observed a higher distribution of SNP density in intergenic regions compared with other regions of methylated $\mathrm{CpG}$ sites at all methylation levels. Here, intergenic regions are defined as regions without any gene annotation. This result was expected, as most mutations in intergenic regions are not subject to selection, and thus, display a higher mutation rate. Second, compared with intergenic regions, the mutation rates in other genomic regions (e.g., intronic regions, exonic regions, promoter regions, and CGIs) were moderately lower than those of the whole genome regardless of their methylation level. Third, for those CpG sites located in the CGIs or promoter regions, the mutation rate was overall very low, but consistent, regardless of the methylation level. This trend is likely due to the lack of methylation and the suppression of methylcytosine deamination in CGIs and promoter regions [4]. Finally, the CpG sites in intergenic regions and intronic regions showed a higher mutation rate with low-intermediate and intermediate levels of methylation.

\section{Mutation rate of methylated CpG site among chromosomes}

We further examined the mutation rate pattern in human chromosomes (Figure 3). It is already known that the mutation rate varies among chromosomes. Within chromosomes, we observed that CpG sites with lowintermediate and intermediate levels of methylation had a higher mutation rate in all autosomes compared with that of other methylated CpG sites. Furthermore, there were differences in the mutation rate among autosomes. For example, methylated CpG sites in chromosomes 4, 13,18 , and 21 had a higher mutation rate compared with that in other autosomes. We noticed that these four autosomes have overall low gene densities; thus, we further analyzed the relationship between the mutation rate and gene density. We found a negative correlation between the mutation rate and gene density (see additional file 4), and this result further supports the higher mutation rate at the intergenic regions.

A similar mutation pattern could not be observed in the $\mathrm{X}$ chromosome due to an overall low mutation rate in this sex chromosome. We only found a slightly higher mutation rate in the $\mathrm{CpG}$ sites with a lowly methylated level (0-20\%) (Figure 3).

\section{Mutation rate of non-CpG methylated cytosines}

The $\mathrm{H} 1$ cell line has abundant non- $\mathrm{CpG}$ methylation ( $\mathrm{mCHG}$ and $\mathrm{mCHH}$, where $\mathrm{H}=\mathrm{A}, \mathrm{C}$ or $\mathrm{T}$ ) that accounted for nearly $25 \%$ of all methylated cytosines. Therefore, we investigated the mutation rate in these methylated non- 


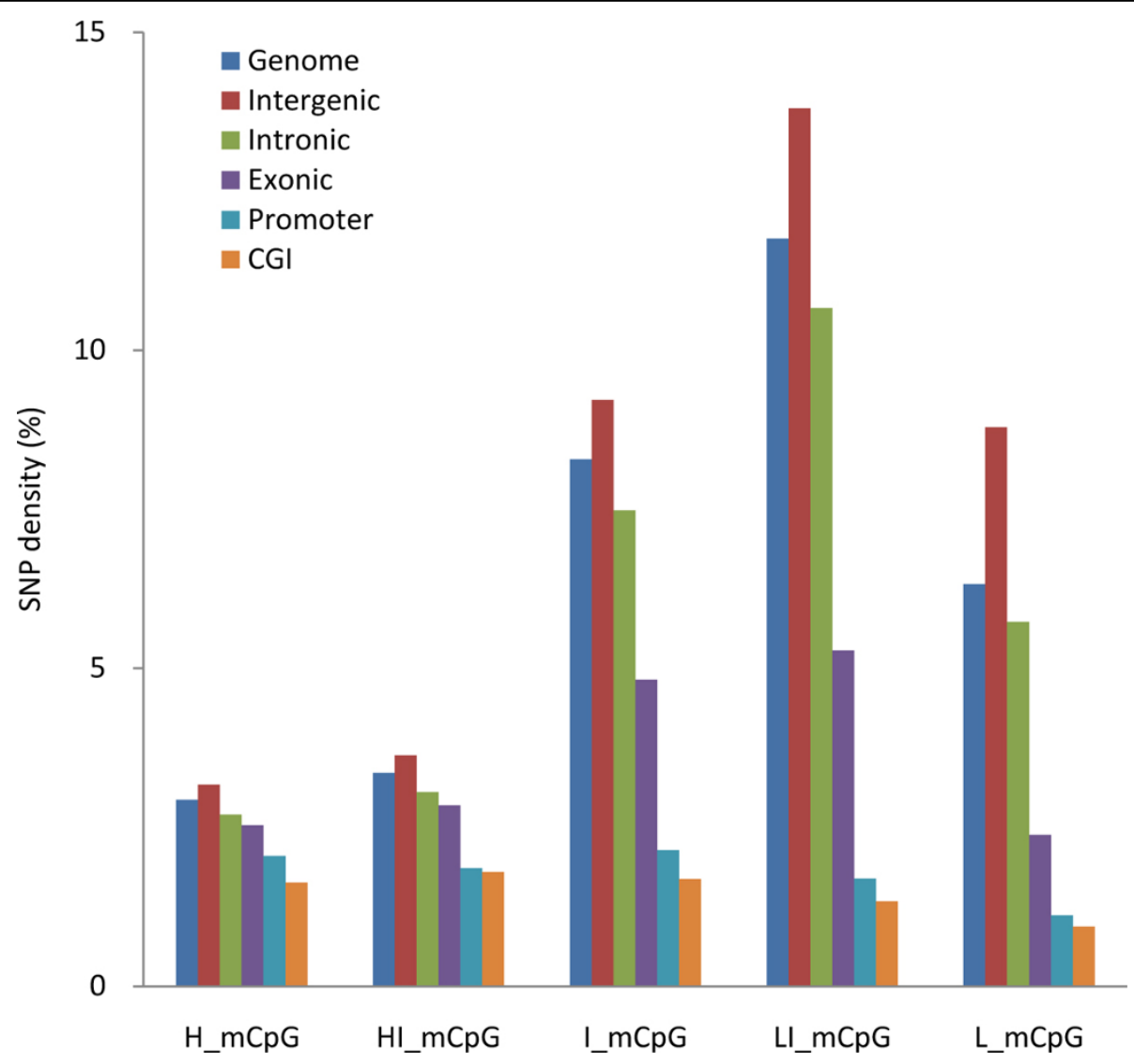

Figure 2 The mutation rates for methylated $\mathrm{CpG}$ sites in the human genome and categorized genomic regions. The $X$-axis includes $\mathrm{CpG}$ sites with different methylation levels (H_mCpG: highly methylated CpG (> 80\%); HI_mCpG: high-intermediately methylated CpG (60-80\%); I_mCpG: intermediately methylated CpG (40-60\%); LI_mCpG: low-intermediately methylated CpG (20-40\%); L_mCpG: lowly methylated CpG ( $\leq 20 \%)$ ). The Y-axis is the mutation rate that was calculated as the density of SNPs at CpG sites.

CpG sites. A summary of the mutation rate from $\mathrm{mCHG}$ and $\mathrm{mCHH}$ sites was provided in additional file 5. We found that the mutation rates in methylated non-CpG sites were low across the different methylation levels in these sites (0.38-0.42\% for $\mathrm{mCHG}$, and 0.39-0.54\% for $\mathrm{mCHH}$ ); thus, we did not observe the pattern as in the methylated CpG sites.

\section{Sequence divergence at methylated $\mathrm{CpG}$ sites}

To further confirm our observations based on SNP density, we used human-chimpanzee sequence divergence to infer the mutation rate (Figure 4). Again, we found the highly methylated CpG sites (9.94\%) had larger divergence than lowly methylated CpG sites (7.23\%). More interestingly, the divergence of CpGs with methylation levels of $20-40 \%$ and $40-60 \%$ was $10.66 \%$ and $10.49 \%$, respectively. This divergence was higher than the divergence of CpGs with methylation levels of $60-80 \%$ and $80-100 \%$, which is consistent with our SNP density data. To exclude the possible effects of selection on functional regions, we further investigated the divergence in intergenic regions. A similar, but even more evident, pattern was observed (see additional file 6).

\section{Allele frequency at methylated CpG sites}

Using CEU population data from the HapMap Project (release 21), we assigned an allele frequency for each SNP. After mapping C/A, C/G, and C/T SNPs to methylated CpG sites, the ancestor allele of a methylated cytosine site could be inferred by comparing a SNP with its chimpanzee ancestral allele based on our previous method [4]. There were 250,541 methylated CpG sites with inferred ancestor alleles. By calculating the correlation between the methylation level and allele frequency in these methylated CpG sites, we found a significant Pearson correlation between methylation level and cytosine allele frequency $(r=0.426, P<2.2 \mathrm{E}-16)$ as compared to the correlation between methylation level and major allele frequency $(r=0.198, P<2.2 \mathrm{E}-16)$ or the correlation between methylation level and ancestor allele frequency $(r=0.359, P<2.2 \mathrm{E}-16)$. We further binned CpG sites into five classes according to cytosine allele frequency 


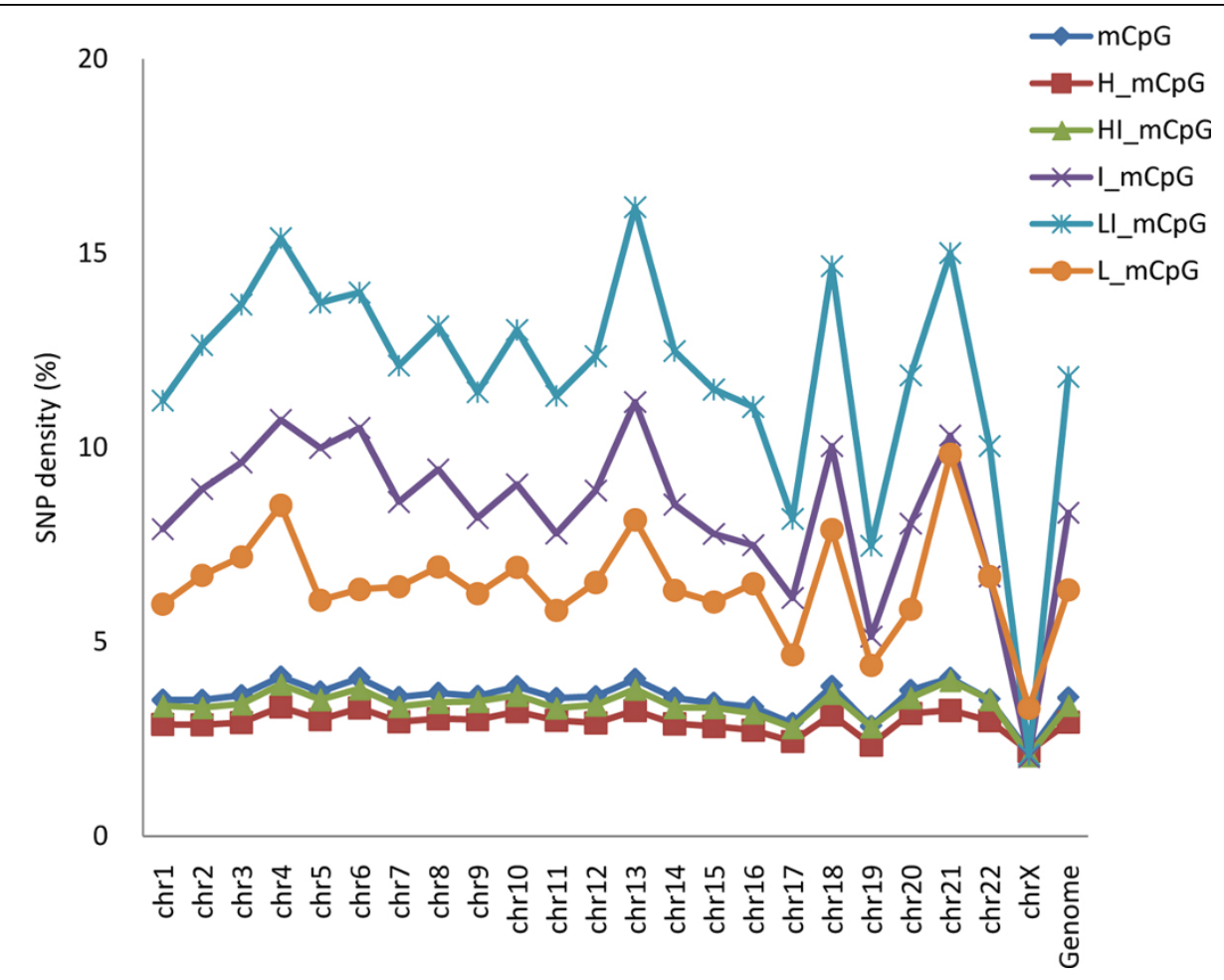

Figure 3 Comparison of the mutation rates at methylated CpG sites in the human genome and its chromosomes. Refer to Figure 1 legend for interpretation of abbreviations.

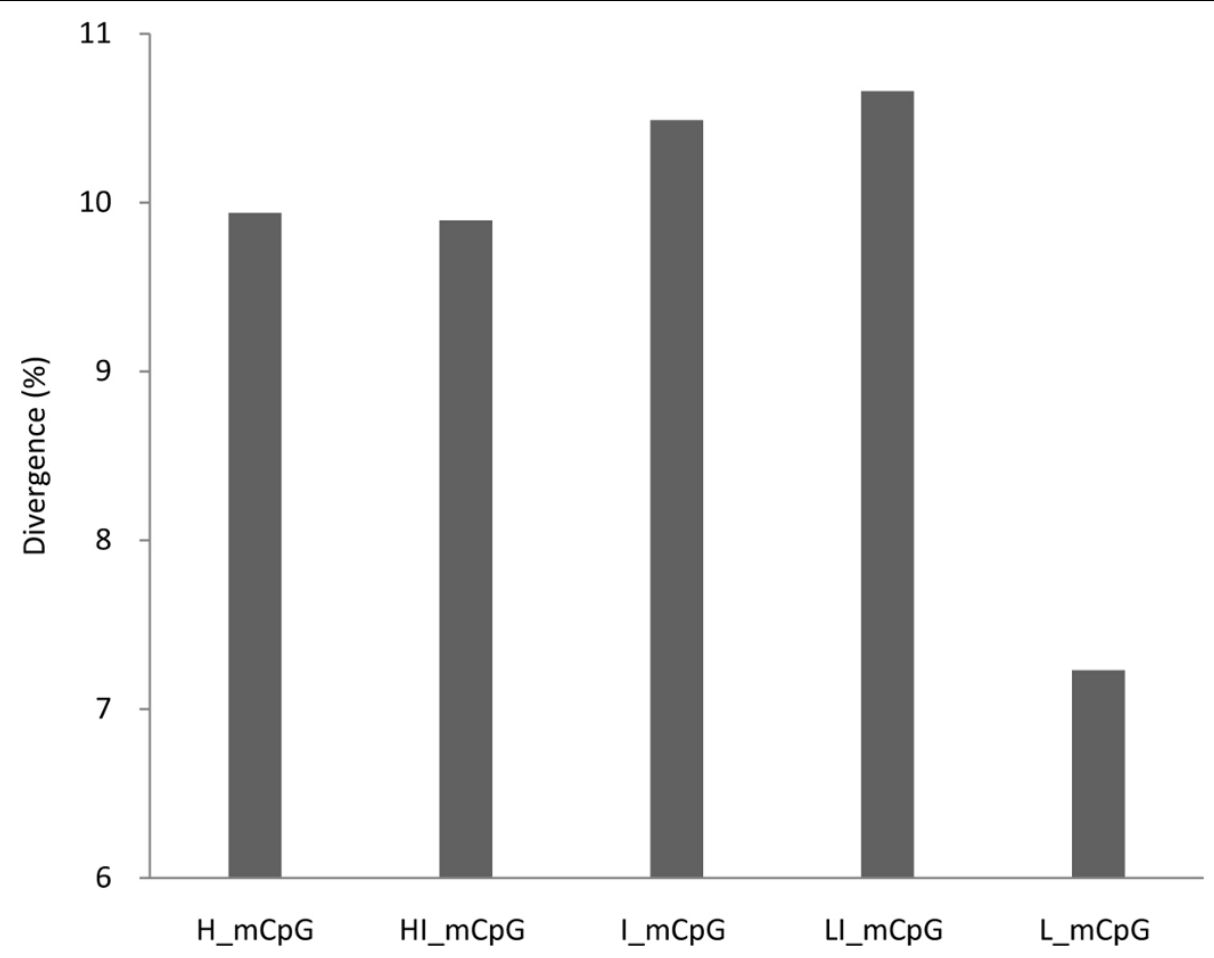

Figure 4 Human-chimpanzee divergence in CpG sites categorized by different methylation level. Refer to Figure 1 legend for interpretation of abbreviations. 
and carried out a regression analysis using methylaton level data. The methylation level associated with the cytosine allele frequency displayed a strong linear dependence with a correlation coefficient $r=0.977$ (Figure 5).

To further test whether the methyltion level depends on the allele frequency information, we examined the methylation level in CpG sites with different allele frequencies. First, we compared the methylation level when the major allele is cytosine with that when it is not. The methylation level was higher with cytosine as the major allele than with other bases as the major allele (0.798 vs. $0.605, P<2.2 \mathrm{E}-16)$. The methylation level was also higher when the ancestral allele is cytosine than other bases ( 0.792 vs. $0.703, P<2.2 \mathrm{E}-16)$. The directionality observed in these values is consistent with the fact that deamination of methylated cytosine results in $\mathrm{C} \rightarrow \mathrm{T}$ mutations that likely remain unrepaired and, therefore, become polymorphisms. For those alleles with cytosine as the ancestral allele, we further categorized them as to whether their current major allele is cytosine or other base. We found that for those alleles with cytosine as the ancestor, their methylation level significantly increased when cytosine became the current major allele relative to another base as the current major allele ( 0.810 vs. 0.610 , $P<2.2$ E-16). The increased methylation level of $\mathrm{CpG}$ sites with cytosine as both the ancestral allele and the current major allele further suggest that these methylated
CpGs are representative of hypermutable sites. Taking together, these results indicated that the subset of methylated CpGs in the derived allele dataset is not a random subset of the genome. The observed overrepresentation of methylated CpGs in all data sets suggests that the methylated CpGs variable is indicative of the previously known hypermutability of methylated cytosines.

\section{Conclusion}

Previous studies have suggested that methylated CpG sites correlate strongly to a high rate of mutation. However, whether and how the higher methylation level causes the higher mutation rate is largely unknown. Our analysis showed that CpG sites with low-intermediate to intermediate levels of methylation (20-60\%) actually had a much higher mutation rate as reflected in recent human nucleotide diversity as compared with CpG sites with other methylation levels at different scales, including the categorized genomic regions, whole chromosome, and the whole genome. Consistently, we observed a similar pattern by using human-chimpanzee divergence data.

We further examined variation in the mutation rate among chromosomes. Within autosomal chromosomes, we observed that $\mathrm{CpG}$ sites with low-intermediate and intermediate levels of methylation had a higher mutation rate compared with $\mathrm{CpG}$ sites with other methylation levels. We found that autosomes with low gene density

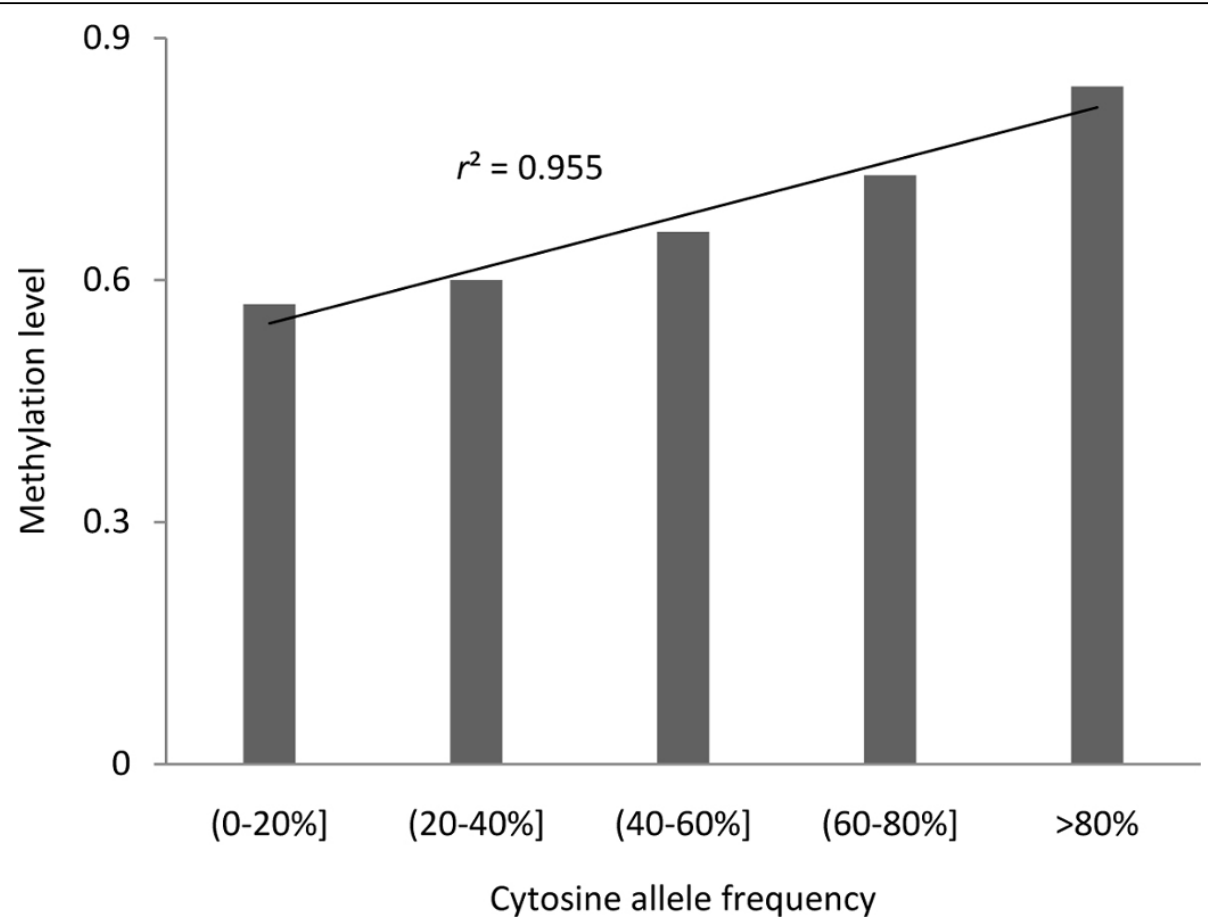

Figure 5 The correlation between methylation level and cytosine allele frequency. The $X$-axis includes five classes of $C p G$ sites defined on the basis of their cytosine allele frequency. The $Y$-axis is the methylation level that was computed as the number of methylated reads over the total number of reads mapped to each cytosine base. The correlation coefficient $\left(r^{2}\right)$ is shown on the plot. 
tend to have an overall higher mutation rate. Because of male-driven evolution [27] in humans, mutation rates are typically highest on the $\mathrm{Y}$ chromosome, intermediate on autosomes, and lowest on the $\mathrm{X}$ chromosome. Indeed, the mutation rate was higher on the $\mathrm{CpG}$ sites in autosomes than that in the $\mathrm{X}$ chromosome. However, we could not estimate the mutation rate in the $\mathrm{Y}$ chromosome due to the overall low quality data. Further investigation is necessary when high quality data in this sex chromosome is available.

In summary, our study found a significant correlation between the extent of germ line methylation and the mutation rate at human CpG sites. Specifically, we found that there is a high mutation rate in low-intermediately to intermediately methylated CpG sites at different scales, from the categorized genomic region, whole chromosome, to the whole genome level. Mutation rate has been found to be correlated with proximal DNA methylation patterns [11,28], local GC content[10], local sequence lengths and genomic regions[7], local or regional nucleotide composition, crossover rate, distance to telomeres, chromatin compaction [24], as well as the replication timing [2]. Our analysis provides the first supportive evidence regarding the mutation rate variation at human methylated CpG sites using genome-wide single base resolution methylation data. However, further integrative investigation of genetic factors on the mutation rate and methylation level is warranted. Finally, we have shown that there is a significant correlation between methylation level and cytosine allele frequency in the human genome.

\section{Additional material}

Additional file 1: The mutation rates at CpG sites inH1 and IMR90 cell lines. Refer to Figure 1 legend for interpretation of abbreviation.

Additional file 2: The mutation rates for all CpG sites, methylated CpG sites and unmethylated CpG sites in different DNA strands. Refer to Figure 1 legend for interpretation of abbreviation.

Additional file 3: The mutation rates for methylated CpG sites that are covered by different levels of sequencing reads. Refer to Figure 1 legend for interpretation of abbreviation.

Additional file 4: The correlation between mutation rate and gene density for methylated CpG sites at the chromosome level. Each chromosome is plotted as a dot with its gene density per megabase and SNP density on the $X$ and $Y$ axis, respectively. The correlation coefficient $\left(r^{2}\right)$ is shown on the plot.

Additional file 5: The mutation rates for $\mathrm{CHG}$ and $\mathrm{CHH}$ sites with different methylation levels. The $\mathrm{X}$-axis includes $\mathrm{CHH}$ and $\mathrm{CHG}$ sites with different methylation levels (mC: methylated site; $\mathrm{H} \_m C$ : highly methylated site (> 80\%); HI_mC: high-intermediately methylated site (6080\%); I_mC: intermediately methylated site (40-60\%); LI_mC: lowintermediately methylated site (20-40\%); L_mC: lowly methylated site ( $20 \%)$ ). The $\mathrm{Y}$-axis is the mutation rate that was calculated as the density of SNPs at $\mathrm{CHH}$ and $\mathrm{CHG}$ sites.

Additional file 6: Human-chimpanzee divergence for methylated CpG sites in the human genome and intergenic regions. Refer to Figure 1 legend for interpretation of abbreviation.

\section{Acknowledgements}

We thank members of the Bioinformatics and Systems Medicine Laboratory for their useful discussion and suggestions. We also thank Rebecca Hiller Posey for her assistance with polishing the manuscript.

This article has been published as part of BMC Genomics Volume 13 Supplement 8, 2012: Proceedings of The International Conference on Intelligent Biology and Medicine (ICIBM): Genomics. The full contents of the supplement are available online at http://www.biomedcentral.com/ bmcgenomics/supplements/13/S8.

\section{Author details}

${ }^{1}$ Department of Biomedical Informatics, Vanderbilt University School of Medicine, Nashville, TN 37232, USA. ²Department of Psychiatry, Vanderbilt University School of Medicine, Nashville, TN 37232, USA. ${ }^{3}$ Department of Cancer Biology, Vanderbilt University School of Medicine, Nashville, TN 37232, USA. ${ }^{4}$ Center for Quantitative Sciences, Vanderbilt University Medical Center, Nashville, TN 37232, USA.

\section{Authors' contributions}

JX carried out the data analysis and drafted the manuscript. LH designed the project, carried out the data analysis, and participated in drafting the manuscript. ZZ conceived of the study, designed the project, and participated in drafting the manuscript. All authors read and approved the final manuscript.

\section{Competing interests}

The authors declare that they have no competing interests.

Published: 17 December 2012

\section{References}

1. Hodgkinson A, Eyre-Walker A: Variation in the mutation rate across mammalian genomes. Nat Rev Genet 2011, 12:756-766.

2. Stamatoyannopoulos JA, Adzhubei I, Thurman RE, Kryukov GV, Mirkin SM, Sunyaev SR: Human mutation rate associated with DNA replication timing. Nat Genet 2009, 41:393-395.

3. Duret L, Arndt PF: The impact of recombination on nucleotide substitutions in the human genome. PLoS Genet 2008, 4:e1000071.

4. Jiang C, Zhao Z: Mutational spectrum in the recent human genome inferred by single nucleotide polymorphisms. Genomics 2006, 88:527-534.

5. Ehrlich M, Norris KF, Wang RY, Kuo KC, Gehrke CW: DNA cytosine methylation and heat-induced deamination. BiosC Rep 1986, 6:387-393.

6. Jiang C, Han L, Su B, Li WH, Zhao Z: Features and trend of loss of promoter-associated CpG islands in the human and mouse genomes. Mol Biol Evol 2007, 24:1991-2000.

7. Zhao Z, Jiang C: Methylation-dependent transition rates are dependent on local sequence lengths and genomic regions. Mol Biol Evol 2007, 24:23-25.

8. Han L, Su B, Li WH, Zhao Z: CpG island density and its correlations with genomic features in mammalian genomes. Genome Biol 2008, 9:R79.

9. Cooper DN, Youssoufian H: The CpG dinucleotide and human genetic disease. Hum Genet 1988, 78:151-155.

10. Fryxell KJ, Moon WJ: CpG mutation rates in the human genome are highly dependent on local GC content. Mol Biol Evol 2005, 22:650-658.

11. Mugal CF, Ellegren $\mathrm{H}$ : Substitution rate variation at human $\mathrm{CpG}$ sites correlates with non-CpG divergence, methylation level and GC content. Genome Biol 2011, 12:R58.

12. Down TA, Rakyan VK, Turner DJ, Flicek P, Li H, Kulesha E, Gräf S, Johnson N, Herrero J, Tomazou EM: A Bayesian deconvolution strategy for immunoprecipitation-based DNA methylome analysis. Nat Biotechnol 2008, 26:779-785.

13. Lister R, Pelizzola M, Dowen RH, Hawkins RD, Hon G, Tonti-Filippini J, Nery JR, Lee L, Ye Z, Ngo QM: Human DNA methylomes at base resolution show widespread epigenomic differences. Nature 2009, 462:315-322.

14. Du X, Han L, Guo AY, Zhao Z: Features of methylation and gene expression in the promoter-associated CpG islands using human methylome data. Comp Funct Genomics 2012, 2012:598987.

15. Su Z, Xia J, Zhao Z: Do microRNAs preferentially target the genes with low DNA methylation level at the promoter region? Lect Notes Comput Sci 2012, 6840:253-258. 
16. Su Z, Xia J, Zhao Z: Functional complementation between transcriptional methylation regulation and post-transcriptional microRNA regulation in the human genome. BMC Genomics 2011, 12:S15.

17. Su Z, Han L, Zhao Z: Conservation and divergence of DNA methylation in eukaryotes: new insights from single base-resolution DNA methylomes. Epigenetics 2011, 6:134-140

18. National Center for Biotechnology Information. [http://www.ncbi.nlm.nih. gov/].

19. ENSEMBL Database. [ftp://ftp.ensembl.org/pub]

20. Han L, Zhao Z: Contrast features of $\mathrm{CpG}$ islands in the promoter and other regions in the dog genome. Genomics 2009, 94:117-124.

21. Zhao Z, Han L: CpG islands: algorithms and applications in methylation studies. Biochem Biophys Res Commun 2009, 382:643-645

22. Han L, Zhao Z: CpG islands or CpG clusters: how to identify functional GC-rich regions in a genome? BMC Bioinformatics 2009, 10:65.

23. Takai $\mathrm{D}$, Jones $\mathrm{PA}$ : Comprehensive analysis of $\mathrm{CpG}$ islands in human chromosomes 21 and 22. Proc Natl Acad Sci USA 2002, 99:3740-3745.

24. Chen CL, Rappailles A, Duquenne L, Huvet M, Guilbaud G, Farinelli L. Audit B, d'Aubenton-Carafa Y, Arneodo A, Hyrien O: Impact of replication timing on non-CpG and $\mathrm{CpG}$ substitution rates in mammalian genomes. Genome Res 2010, 20:447-457.

25. UCSC Genome Browser. [http://genome.ucsc.edu/].

26. Reya T, Morrison S, Clarke M, Weissman I: Stem cells, cancer, and cancer stem cells. Nature 2001, 414:105-111.

27. Li WH, Yi S, Makova K: Male-driven evolution. Curr Opin Genet Dev 2002, 12:650-656.

28. Morishita S, Ou W, Hashimoto S, Shimada A, Nakatani Y, Saito TL, Ogoshi K, Matsushima K, Suzuki Y, Sugano S: Genome-wide genetic variations are highly correlated with proximal DNA methylation patterns. Genome Res 2012, 22:1419-1425, .

doi:10.1186/1471-2164-13-S8-S7

Cite this article as: Xia et al:: Investigating the relationship of DNA methylation with mutation rate and allele frequency in the human genome. BMC Genomics 2012 13(Suppl 8):S7.

\section{Submit your next manuscript to BioMed Central} and take full advantage of:

- Convenient online submission

- Thorough peer review

- No space constraints or color figure charges

- Immediate publication on acceptance

- Inclusion in PubMed, CAS, Scopus and Google Scholar

- Research which is freely available for redistribution

Submit your manuscript at www.biomedcentral.com/submit
Biomed Central 